\title{
The world of communication: non-verbal communication (NVC), neuro-linguistic programming (NLP) and persuasion strategies
}

\author{
Il mondo della comunicazione: comunicazione non verbale (CNV), \\ programmazione neuro-linguistica (PNL) e strategie di persuasione \\ El mundo de la comunicación: comunicación no verbal (CNV), \\ programación neurolingüística (PNL) y estrategias de persuasión
}

\author{
Noemi Martini, ${ }^{1}$ Vincenzo M. Mastronardi ${ }^{2}$ \\ ${ }^{1}$ Dottoressa Magistrale in Investigazione, Criminalità e Sicurezza Internazionale. Università degli Studi Internazionali \\ di Roma (UNINT); ${ }^{2}$ Psichiatra Criminologo clinico Docente Sapienza Università di Roma e Università degli Studi \\ Internazionali di Roma, Italy
}

\begin{abstract}
What are the forms, methods and characteristics of communication? Communication, understood as the exchange of information from an issuer to a recipient, comes into contact with all the social and cultural spheres belonging to it and is focused here to show the main markers that characterize it. The work is based on an analysis of all those aspects that can influence the communication exchange or even distort the meaning of the message sent. The research questions were also addressed to the fascinating field of non-verbal communication and the characteristics of body gestures, understood as distinctive signals with a strong social impact, also and above all in police interviews and interrogations. Particular reserve was addressed to the many years of experience of Michael Napier, special agent of the prestigious Quantico FBI Behavioral Sciences Unit, for his meticulous work on the aspects necessary in the proper execution of an investigative investigation. The work ends with a current reflection and deepening on the techniques of persuasion and manipulation of communication, which can become a real weapon to strike, on the one hand positively convincing and working on self-esteem and to help individuals achieve their own objectives, on the other hand by manipulating and misinforming a large slice of the population with psychological, political and digital tools.
\end{abstract}

\section{RIASSUNTO}

Quali sono le forme, le modalità e le caratteristiche della comunicazione? La comunicazione, intesa come scambio di informazioni da un emittente ad un destinatario, entra a contatto con tutti gli ambiti sociali e culturali ad essa appartenenti e viene qui messa a fuoco per mostrare i principiali marcatori che la caratterizzano. Il lavoro si basa su un'analisi di tutti quegli aspetti che possono influenzare lo scambio comunicativo o addirittura distorcere il significato del messaggio inviato. Le domande di ricerca sono state rivolte anche all'affascinante campo della comunicazione non verbale e alle caratteristiche dei gesti corporei, intesi come segnali distintivi e di forte impatto sociale, anche e soprattutto nei colloqui e interrogatori di polizia. Particolare riserbo è stato rivolto all'esperienza pluriennale di Michael Napier, agente speciale della prestigiosa Unità di Scienze Comportamentali dell'FBI di Quantico, per il suo meticoloso lavoro sugli aspetti necessari in una corretta esecuzione di un'indagine investigativa. Il lavoro si conclude con un'attuale riflessione e approfondimento sulle tecniche di persuasione e manipolazione della comunicazione, che possono diventare una vera e propria arma per colpire, da una parte positivamente convincendo e lavorando sull'autostima e per aiutare gli individui a raggiungere i propri obiettivi, dall'altra manipolando e disinformando un'ampia fetta di popolazione con strumenti psicologici, politici e digitali.

\begin{abstract}
RESUMEN
¿Cuáles son las formas, métodos y características de la comunicación? La comunicación, entendida como el intercambio de información de un emisor a un receptor, entra en contacto con todos los ámbitos sociales y culturales que le pertenecen y se enfoca aquí para mostrar los principales marcadores que la caracterizan. El trabajo se basa en un análisis de todos aquellos aspectos que pueden influir en el intercambio comunicativo o incluso distorsionar el significado del mensaje enviado. Las preguntas de investigación también se dirigieron al fascinante campo de la comunicación no verbal y las características de los gestos corporales, entendidos como señales distintivas de fuerte impacto social, también y sobre todo en las entrevistas e interrogatorios policiales. Particular reserva se dirigió a los muchos años de experiencia de Michael Napier, agente especial de la prestigiosa Unidad de Ciencias del Comportamiento Quantico FBI, por su minucioso trabajo sobre los aspectos necesarios para la correcta ejecución de una investigación investigativa. El trabajo finaliza con una reflexión actual y un análisis en profundidad sobre las técnicas de persuasión y manipulación de la comunicación, que pueden convertirse en un arma real para golpear, por un lado convincente positivamente y trabajando en la autoestima y para ayudar a las personas a alcanzar la suya propia. objetivos, por otro lado, manipulando y desinformando a una gran parte de la población con herramientas psicológicas, políticas y digitales.
\end{abstract}




\section{La comunicazione e la sfera non verbale}

Il concetto di comunicazione è racchiuso nella natura umana perché l'uomo comunica idee, messaggi, stati d'animo ed emozioni, consapevolmente e inconsapevolmente, ed è proprio grazie alla comunicazione tra esseri viventi che si è sviluppata la specie umana e conseguentemente la società civile.

Volendo introdurre un argomento così ampio, è opportuno partire da una semplice definizione, "comunicazione" deriva dal latino communicatio -onis - mettere in comune. Infatti, viene considerata come quel processo e le modalità di trasmissione di un'informazione da un individuo ad un altro, attraverso lo scambio di un messaggio elaborato secondo le regole di un determinato codice. Ma per poter comunicare è necessario che si verifichino le seguenti condizioni di base:

- due o più soggetti devono entrare in contatto tra di loro in modo anche indiretto,

- devono esistere dei messaggi da trasmettere;

- deve esistere un codice comune tra i soggetti che permetta la comprensione del messaggio;

- deve sussistere la volontà di comunicare.

L'essere umano, infatti, sin dagli albori, ha cercato di comunicare e di trasmettere un segnale del suo passaggio, proprio perché la comunicazione è alla base di tutto quello che l'uomo ha potuto conoscere e realizzare. Dapprima, con gesti e vocalizzi e successivamente con disegni e incisioni nelle caverne, arrivando così alle prime forme di scrittura nei papiri, all'invenzione del codice alfabetico e numerico scritto su carta. Inoltre, questo progresso è stato favorito dal contributo di vari strumenti e discipline come la musica, il cinema, la televisione e i giornali, fino ad arrivare oggi, a forme più complesse di comunicazione digitale.

"La comunicazione è scambio simbolico, in cui intervengono in modo attivo e creativo due soggetti o più. Non c'è asimmetria tra emittente e ricevente, ma reciprocità: l'uno e l'altro sono attori allo stesso titolo del processo comunicativo. In gioco non c'è soltanto la produzione/trasmissione di un messaggio, bensì la costruzione del suo significato" (Lever F., Rivoltella P.C., Zanacchi A., 2020).

Il soggetto umano vive all'interno di una rete di comunicazioni interpersonali che non possono prescindere dai contesti sociali, storici e culturali; non esiste comunicazione che non risenta di questi contesti. Si è ritenuto opportuno, quindi, produrre un quadro generale in grado di rendere più accessibile il vasto mondo della comunicazione. Dal semplice studio della parola, alle varie definizioni del dizionario, agli studi dei molteplici autori che si sono interessati all'argomento, fino alle varie categorie e tipologie di linguaggio impiegate quotidianamente. Pur essendo un tema così banale, gli aspetti che vengono dati per scontati e quelli ancora da scoprire sono molteplici, ma sostanzialmente la comunicazione è l'azione più utilizzata dall'uomo. Impiegandola involontariamente, perché appresa durante l'infanzia, ed essendo articolata in una molteplicità di aspetti impliciti, che si manifestano quotidianamente, non viene adeguatamente studiata e compresa, questo lavoro invece cerca di analizzarla e comprenderla a fondo.

La comunicazione verbale, che impiega sia il linguaggio orale ma anche quello scritto, è la modalità di comunicazione forse più usata e diffusa fra gli esseri umani. È utilizzata per semplificare la realtà, per organizzarla, per analizzarla ma anche per ricordare e tramandare le esperienze passate alle nuove generazioni o per proiettarle nel futuro. La comunicazione verbale è costituita dalle parole, usate nel parlato e nello scritto, e normalmente è anche il livello di cui l'uomo ne è più consapevole e quindi viene curata maggiormente. Infatti, per esprimersi l'essere umano sceglie le parole con cura e le adatta al registro personale, in base all'inter- locutore che le si prospetta. In ogni caso, cerca di inquadrare il discorso in base e in modo che sia il più comprensibile possibile, provando ad essere maggiormente persuasivo per manifestare nell'interlocutore il massimo interesse possibile.

Il linguaggio verbale può svolgere varie funzioni:

- Funzione ideativa: permette di esprimere, in uno scambio comunicativo con altri, le proprie esperienze e idee, di condividere le proprie conoscenze, di scambiare informazioni sulla realtà in cui l'uomo vive e agisce;

- Funzione interpersonale: permette agli individui di interagire con gli altri, provocare e stimolare le loro reazioni, creando un continuo feedback, carico di connotazioni empatiche e affettive;

- Funzione educativa: l'educazione come sviluppo e formazione di conoscenze mentali, sociali e culturali si basa, in primis, sull'interazione verbale, infatti, le conoscenze vengono trasmesse attraverso comunicazioni orali e scritte, sia per conoscere la realtà circostante, sia per risolvere in modo più efficace i problemi;

- Funzione testuale: consente di stabilire legami fra le varie parti di un messaggio e il contesto in cui esso si è manifestato. Il linguaggio è un insieme di segni dotati di significato, esso porta a favorire il ragionamento, la riflessione e lo sviluppo del pensiero logico;

- Funzione simbolica: permette all'individuo di esprimersi tramite i simboli. Esso può rappresentare un oggetto, una persona, una situazione mediante un simbolo verbale, che si trasforma in un legame psicologicamente necessario per indicare quell'oggetto, quella persona o quella situazione.

La comunicazione è quindi un processo di interazione sociale che permette di scambiare messaggi da un emittente ad un ricevente tramite un canale comunicativo, o medium, che è il mezzo tramite per inviare il messaggio. Questo messaggio avrà un codice, verbale o non verbale, che esprime la sfera sentimentale ed affettiva dell'individuo che lo manda. Chi emette un messaggio deve possedere una certa credibilità entro un determinato pubblico e utilizzare la persuasione per far sì che il messaggio sia accolto favorevolmente, perché, non sempre, lo scambio comunicativo va a buon fine, potrebbe essere mal interpretato o distorto in base alla condizione in cui viene trasmesso.

Un ottimo esempio per schematizzare il processo comunicativo è stato fornito da Roman Jakobson, linguista e semiologo russo, è considerato uno dei principali fondatori della scuola del formalismo e dello strutturalismo, ma soprattutto uno dei più importanti linguisti del XX secolo. A lui si deve la "teoria della comunicazione linguistica" dove per primo ha suddiviso in sei gli elementi essenziali per la buona riuscita di uno scambio comunicativo.

Analizzata in uno schema come questo (Fig. 1) può sembrare

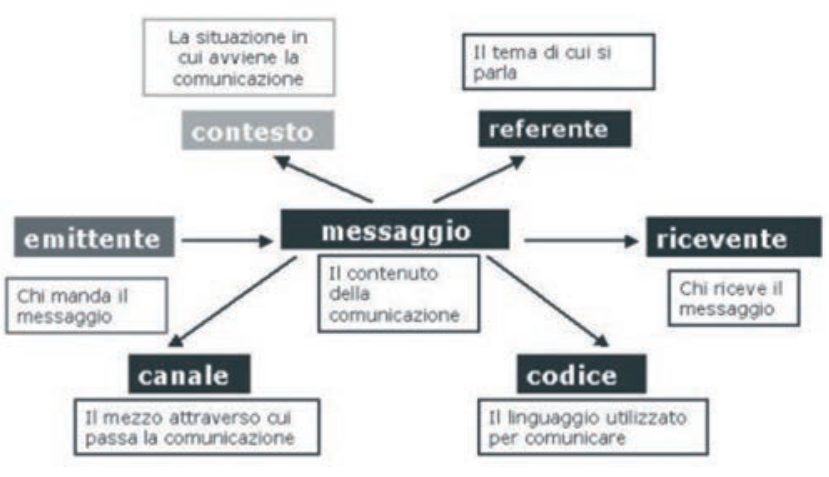

Fig. 1. Teoria della comunicazione linguistica di R. Jakobson. 
elementare codificare il messaggio che viene trasmesso durante la conversazione, ma tralasciamo tutto l'affascinante mondo dei movimenti, dei gesti corporei, delle espressioni che verosimilmente comunicano, molto più delle parole. Quindi non si può non comunicare (Watzlawich P., 1971), perché la comunicazione è insita nella vita e tutti i comportamenti umani sono una forma di comunicazione. Anche il silenzio è comunicazione, anzi, in certe occasioni può trasmettere messaggi molto forti e intensi.

Approfonditi gli aspetti di comunicazione non verbale (CNV) - aspetto esteriore, spazio interpersonale, espressioni del viso, sistema paralinguistico, codice olfattivo - si nota come questi siano mezzi e tramiti per inviare un messaggio, molto spesso confuso o non preciso, ma se ben interpretato e studiato potrebbe diventare uno strumento molto più intenso del linguaggio verbale.

L'ampio mondo della comunicazione non verbale completa o a volte contraddice il linguaggio espresso verbalmente. È dunque un linguaggio "di relazione" e comprende la postura, i movimenti, la posizione occupata dalla persona nello spazio, l'abbigliamento. Essa funge da canale di dispersione, in quanto, essendo meno facile da controllare rispetto alla comunicazione verbale, lascia filtrare aspetti e contenuti molto più profondi. Il linguaggio non verbale può esistere anche in assenza di comunicazione verbale, poiché, in alcuni contesti, può trasmettere messaggi altrettanto significativi e pieni di contenuto. Il linguaggio e i codici non verbali, infatti, costituiscono un sistema affascinante ed eterogeneo attraverso cui è possibile creare e condividere il senso e l'essenza del pensiero dei membri di una comunità sociale. I codici non verbali sono:

- Cinesico: studia il linguaggio del corpo,

- Prossemico: studia il comportamento spaziale (Ekman P., 1968),

- Aptico: il processo di riconoscimento degli oggetti attraverso il tatto,

- Cronemico: studia il tempo in cui si verifica un dato movimento,

- Olfattivo: linguaggio che utilizza segni percepiti attraverso il naso, o altri organi sensibili all'odore, ai profumi, alle esalazioni. Questi codici singolarmente o in combinazione tra loro e con il supporto dei componenti linguistici, svolgono finalità fondamentali di comunicazione: produrre e comprendere i messaggi inviati oralmente, esprimere e interpretare le emozioni, creare e comunicare identità personali e sociali, presentare se stessi, gestire le interazioni, definire le relazioni interpersonali, influenzare gli altri, perpetrare o scoprire inganni. "La CNV è un fenomeno interattivo che coinvolge a diversi livelli di intenzionalità e consapevolezza tutti i partecipanti allo scambio comunicativo" (Maricchiolo F., 2017).

Quindi il corpo umano parla, ma non solo con la voce. Tutti i giorni con i gesti del corpo, espressioni delle emozioni, sguardi, regolazione del tono o del ritmo vocale, gesti, posture, abbigliamento, spazio interpersonale, l'uomo trasmette messaggi; ma il linguaggio corporeo è talmente spontaneo che raramente è consapevole di trasmettere forti messaggi con i gesti.

Molto rilevante per la causa è l'attenta osservazione del volto dell'interlocutore. Il viso umano è estremamente espressivo, ci sono ben 36 muscoli per mettere in atto i movimenti e le espressioni facciali e riescono a contribuire nell'esprimere un'ampia varietà di emozioni per avvalorare o contraddire ciò che l'uomo comunica. In particolare, gli occhi sono un organo con un'ampia struttura di terminazioni nevose e circondati da muscoli extra-oculari che ci permettono di contrarsi migliaia di volte al giorno in molti modi diversi. Essi sono ottimi canali di trasmissione di informazioni, dall'interno verso l'esterno, e quindi hanno una forte importanza comunicativa. Indicano, anche, lo stato di eccitazione della persona con cui viene instaurato un dialogo; se questo stato è di origine positiva la pupilla tenderà a dilatarsi, mentre se viene provocato uno stato negativo questa tenderà a restringersi. La dilatazione incontrollata delle pupille trasmetterà anche una funzione seduttiva, quando si è attratti fisicamente da una persona, infatti, questa tenderà a dilatarsi. L'aumento della dimensione pupillare dipende anche dall'attività di problem solving che possiede un soggetto, la dimensione sarà al suo massimo picco quando l'individuo troverà la soluzione ad un problema. Fondamentali saranno i movimenti oculari che possono indicare segnali di "accesso" del messaggio e possono essere di sei/sette tipi con diversi significati da attribuire ad ogni movimento. Nella Fig. 2 vengono descritti e indicati i movimenti oculari e i relativi significati.

Altro esempio di messaggio di CNV è la posizione del corpo all'interno di uno spazio. Ogni persona si muove all'interno di uno spazio, in base ad un sistema di orientamento e di posizionamento rispetto a oggetti o altre persone che lo circondano. La prossemica, come detto precedentemente, è la scienza che studia lo spazio e le distanze come fatto comunicativo. L'antropologo Edward Hall distinse quattro tipi di posizionamento spaziale (Fig. 3):

- Zona intima: dal contatto físico a $45 \mathrm{~cm}$ dal corpo, lo spazio che l'uomo sente come proprio e che "difende"; accessibile solo a chi è più "vicino" dal punto di vista emozionale. Corrisponde allo spazio che intercorre in relazioni intime, in quanto si percepisce l'odore dell'altro, nonché consente di parlare a bassa voce;

- Zona personale: da $45 \mathrm{~cm}$ a 1,20 m, distanza che intercorre tra due persone amiche, che viene adottata nelle relazioni in cui si può toccare l'altro ma stando a debita distanza;

- Zona sociale: da 1,20 m a 3,65 m, distanza che viene mantenuta quando non si conosce l'altra persona, caratteristica delle relazioni più impersonali dove non vi è contatto físico;

- Zona pubblica: da 3,65 $\mathrm{m}$ in poi, è la distanza che intercorre tra un oratore nel palco e un gruppo di persone che lo ascolta.

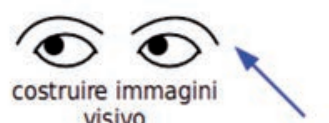
visivo

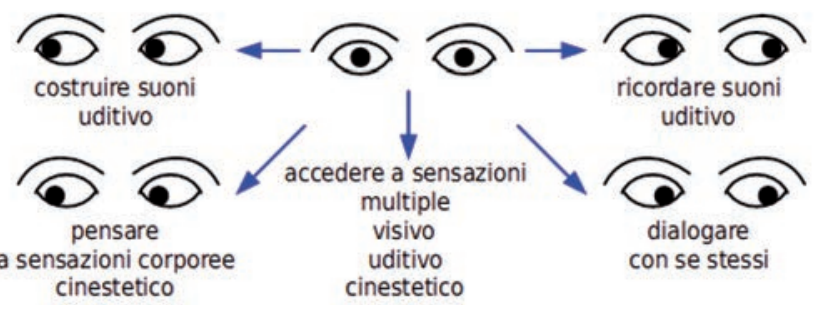

Fig. 2. I movimenti oculari dell'essere umano.

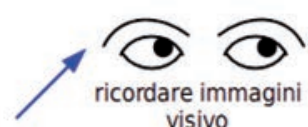
visivo
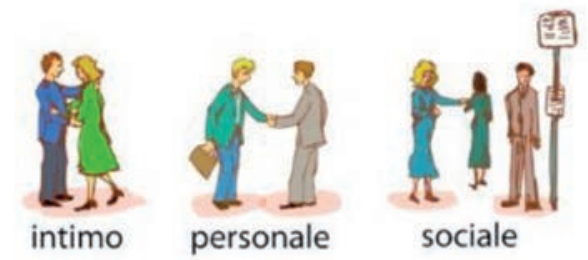
sociale

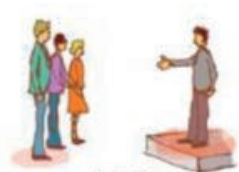

pubblico

Fig. 3. Le distanze sociali di E. Hall. 
Durante una conversazione però queste distanze possono essere soggette a mutamenti, che a loro volta possono fornire altre informazioni: come l'avvicinamento ad una persona può esprimere l'intenzione di iniziare un'interazione oppure, viceversa, l'allontanamento dall'interlocutore potrà segnalare la volontà di interrompere la conversazione. Il contatto fisico, e quindi l'invasione del nostro spazio "personale", può significare rassicurazione o affetto ma anche qui l'aspetto inverso può stare a significare un atteggiamento di minaccia.

Questi sono semplice esempi di come un piccolo movimento degli occhi o il posizionamento del corpo nello spazio possano essere fondamentali durante una conversazione e come questi possano influenzare l'andamento dello scambio comunicativo.

Solo con questa piccola digressione sugli aspetti di CNV si deduce come quest'ultima sia una fonte comunicativa essenziale, probabilmente più potente del linguaggio verbale stesso.

\section{Applicazioni in criminologia e l'esperienza di Michael Napier}

Il comportamento non verbale, più nello specifico, l'analisi facciale e lo studio del linguaggio corporeo hanno portato a risultati utili in ambito criminologico. Infatti lo studio della CNV può essere di ausilio per riconoscere $\mathrm{i}$ segnali di veridicità o falsità delle dichiarazioni rese. Relazionarsi ed interagire con la persona al cospetto dell'agente può far acquisire informazioni che mai potrebbero essere dedotte dalla semplice lettura degli atti documentali, inoltre l'investigatore non deve mai perdere le proprie capacità di comunicazione e dialogo poiché nelle situazioni di emergenza sono queste che gli permettono di agire ed operare in modo corretto, sia per il bene della collettività che nel rispetto di tutte le normative in vigore. Nell'ambito delle attività investigative ed informative la raccolta delle notizie, sia sul possibile autore dell'atto, che sulle dichiarazioni testimoniali, rappresenta un atto fondamentale in quanto permette di riuscire ad accertare la verità sui fatti per cui si indaga, in ambito investigativo e criminologico.

La difficoltà nella raccolta delle informazioni, e talvolta il suo fallimento, derivano dalla presenza di volontà e stati psicologici troppo contrastanti tra l'interrogato e chi interroga. Spesso capita che le persone, di fronte ad una figura autoritaria, se non con la coscienza a posto o se nascondono qualcosa, assumano inconsapevolmente uno stato emotivo reattivo che può facilitare od ostacolare la ricerca della verità. "In particolare, la raccolta di tali informazioni nel corso delle indagini preliminari, risulta un momento fondamentale, in quanto può indirizzare le investigazioni in una direzione anziché in un'altra" (Vitale I., 2019). I segnali che il criminologo guarda in sede di interrogatorio sono fondamentali per interpretare il linguaggio dell'intervistato, ma fondamentale da sottolineare non utilizzabili come prova in tribunale. Il vero problema è che basta un semplice errore di codificazione per incappare in errore, perciò nel processo penale ci vorranno prove certe e fatti concreti per accusare e condannare un soggetto.

Un'altra importantissima funzione della comunicazione non verbale è quella di validare o invalidare i messaggi che derivano dalla comunicazione verbale, anche se viene ritenuta un po' "rozza", nel senso che non è una scienza precisa, la CNV è spontanea, immediata e incapace di mentire. Certe volte, infatti, è possibile mentire, ma non evitare di arrossire o sudare, se l'individuo viene smascherato. Mentre lo stropicciare nervosamente il naso, la bocca, le orecchie con la mano o grattarsi il collo sono generalmente segnali di menzogna.

Mentire per l'interrogato è un atteggiamento che comporta un allestimento di un "lavoro psicologico" ben definito, per creare attendibilità e credibilità davanti all'investigatore. Questo lavoro mentale è in grado di provocare una marcata tensione emotiva, se non un vero e proprio stato d'ansia nell'interrogato. Tale comportamento è gestito con grande sforzo, si devono avere risposte sempre plausibili ed attendibili, coerenti e logiche, non deve contraddirsi mentre espone, ma soprattutto deve controllare le sensazioni, i gesti e i movimenti corporei. Questi aspetti e caratteristiche del corpo e dei suoi movimenti sono tutti indizi che il criminologo noterà e dovrà tenere in considerazione durante l'interrogatorio.

Ma come essere sicuri di ciò che viene interpretato? La comunicazione non verbale va letta con attenzione e serietà, tenendo presente che si tratta di interpretazioni personali che vanno verificate. Un sistema semplice di verifica sono le domande. Un buon comunicatore non è quello che capisce subito cosa la CNV comunica, ma è colui che sa chiedere ed indagare se le sue supposizioni sono corrispondenti a ciò che il suo interlocutore sta vivendo. $\mathrm{Ci}$ sono tre strategie:

1. Domande aperte che invitano ad esprimersi, ad acquisire più elementi senza entrare nel merito della CNV: - "Cosa gliene pare?" - "Cosa intende dire?"

2. Domande chiuse che servono a capire se la CNV è stata interpretata nel modo corretto: - "È arrabbiato, vero?" - "Ho l'impressione che qualcosa non ti vada a genio, è così?" - "Ci sei rimasto male, vero?"

3. Silenzio: è un significativo elemento per capire la situazione. Non parlare ed attendere che sia l'altro ad esprimersi. Cercare elementi di verifica nei comportamenti successivi, nelle azioni che compie, senza intervenire. Per silenzio si può anche intendere una parziale omissione di parole: iniziare una frase ma senza finirla oppure emettere dei suoni di conferma o di attenzione, ma nient'altro, invitando così (obbligando, per certi versi) l'altro a parlare e quindi ad esprimersi di più o meglio.

Quindi non c'è certezza nelle interpretazioni della CNV, non senza opportune verifiche. Questo non significa che sia tutto soggettivo, ma semplicemente, come gli studiosi dimostrano, le persone non sanno leggere la CNV e quindi sbagliano. Se opportunamente allenati, i professionisti possono apprendere la capacità di leggerla in modo efficace, ma resta sempre da considerare il fatto che in sede di interrogatorio le prove ricavate dallo studio della $\mathrm{CNV}$ non sono prove ammissibili in tribunale. Ciò che accade normalmente è che le persone leggono negli altri quello che vogliono leggere o quello che loro vivono, proiettandolo senza rendersene conto, sugli altri. Per questo è importante non solo allenare questa capacità, ma anche conoscere se stessi. È evidente che più una persona si conosce, conosce i propri modi di leggere la realtà, il proprio linguaggio non verbale più è in grado di leggere quella degli altri. La CNV è uno strumento di comunicazione che se considerato seriamente e usato in modo appropriato permette di capire fino in fondo le persone, ma non essendo una prova scientifica non potrà essere utilizzata in fase processuale.

Fondamentale è stato l'intervento di Michael Napier, formatore di agenti speciali per l'FBI di Quantico, con il suo libro "Interviewing and Interrogating Police Style: Practical Police Interviewing" (Napier M. R., 2020). Il suo contributo ha permesso di approfondire tutti quegli aspetti che influenzano un interrogatorio di polizia: dalla personalità dell'intervistato, ai movimenti ed alle espressioni, che non sempre sono utili per capire il sospettato anzi possono trarre in inganno l'agente interrogatore, fino alle caratteristiche che un buon intervistatore deve possedere per risolvere un'indagine. Un agente di polizia nella stanza dell'interrogatorio dovrà indagare ed estrapolare tutti i messaggi che vengono dalle parole, dai gesti o movimenti del soggetto che si trova davanti.

Analizzare il soggetto e carpire tutti gli aspetti del suo carattere 
e comportamento è un lavoro difficile che prevede anni di esperienza sul campo. Ma, in sede di interrogatorio, i messaggi trasmessi dalla $\mathrm{CNV}$ non potranno mai essere considerati delle prove determinanti da portare in tribunale per condannare un sospettato. Saranno solamente "bandiere rosse" (Napier M. R., 2020) che segnalano un comportamento scorretto o deviante, in grado di fornire all'agente interrogatore la possibilità di adattare il proprio atteggiamento nei confronti del sospettato, con l'obiettivo di ricalibrare la strategia iniziale, oppure proseguire infierendo su quegli aspetti considerati più deboli, in modo tale da condurre il sospettato alla confessione. Michael Napier sostiene che tali gesti non dovrebbero essere interpretati come indicatori di inganno, bensì come segnali di stress e disagio, la cui origine dovrebbe essere determinata attraverso la tecnica dell'interrogatorio ravvicinato (al fine di approfondire gli aspetti cruciali). La tecnica migliore è, quindi, quella che evita di trarre conclusioni che possano essere difficili da chiarire finché non si è fatto uno sforzo per determinare le ragioni dei gesti in questione. Non esistono standard nazionali o internazionali per qualificare tale pratica come affidabile, ma è ben noto che le persone leggano nelle situazioni e nelle interazioni personali le proprie opinioni distorte. Questo elemento da solo rende la lettura dei gesti non verbali in cima alla lista delle procedure discutibili e poco sicure. Il contributo di Napier, perciò, ci ha aperto gli occhi sulla possibile fallacia della $\mathrm{CNV}$, per cui essendo una disciplina interpretabile non può essere definita scienza sicura e precisa. Però è possibile sicuramente suddividere questi gesti in due categorie quelli moderatamente sicuri oppure quelli discutibili:

\section{Moderatamente sicuri}

1. Latenza della risposta: lasso di tempo tra la fine di una domanda e l'inizio della risposta. Questo dettaglio aiuta a convalidare la teoria secondo cui un bugiardo esiterà brevemente prima di rispondere a una domanda per determinare se modificare la propria risposta e/o configurare la risposta in modo che soddisfi le informazioni fornite in precedenza.

2. Gesti di copertura: il gesto di coprire la bocca è come se la persona stesse tentando di impedire alle bugie di scappare spingendole di nuovo in gola.

3. Tocco: sembra esserci una tendenza a provare una compulsione a toccare gli occhi, il viso, il naso, il labbro superiore, i lobi delle orecchie, la fronte e/o strofinarsi il viso, specialmente durante una risposta quando si mente;

4. Postura e movimento sulla sedia. Le domande e le risposte in un'intervista o un interrogatorio dovrebbero essere intraprese con entrambe le parti allineate frontalmente, faccia a faccia con uno spazio moderato tra di loro. Come specie, tendiamo a prendere le distanze da cose che offendono o minacciano. Durante l'interrogatorio, una persona che non è offesa o minacciata dalle domande o dalle accuse in genere dovrebbe rimanere faccia a faccia. Il movimento diventa preoccupante quando il sospetto gira il suo corpo da un lato senza muovere la sedia, interrompendo così il contatto visivo in un momento critico. Oppure, può allontanare l'intera sedia dall'allineamento frontale e creare l'allargamento della distanza tra i due, facendo insospettire l'agente interrogatore.

Importante è dunque il posizionamento nella stanza durante l'intervista/interrogatorio. La terminologia usata spesso per questa disposizione dei posti a sedere è "allineamento frontale", che pone le due parti allineate faccia a faccia. Essendo allineato frontalmente, l'agente avrà un percorso chiaro per osservare il movimento degli occhi o del corpo che può richiedere l'interruzione del con- tatto visivo da parte dell'intervistato. Questo segnala che l'argomento in discussione richiede un "interrogatorio ravvicinato" per trovare la causa dell" "occhio sfuggente". Potrebbe essere un gesto molto innocente o potrebbero esserci diversi motivi per l'interruzione del contatto visivo. La posizione di qualcuno che probabilmente dice la verità è solitamente invariata poiché non ha bisogno di agitarsi o muovere la sedia.

\section{Discutibili}

1. Psicopatici: gli psicopatici sono maestri manipolatori e attori senza preoccuparsi di scoprire il suo inganno. Se le caratteristiche di questa persona suggeriscono che è uno psicopatico o un sociopatico, l'ufficiale dovrà essere estremamente cauto nel prendere decisioni basate sul linguaggio del corpo;

2. Barriere: l'origine di molti gesti non verbali deriva dalla nostra intenzione di sopravvivere. L'incrocio di gambe e braccia o la formazione di una barriera posizionando le braccia sul petto, ha lo scopo di coprire la nostra sezione centrale del corpo vulnerabile ad un attacco. La copertura protettiva avviene quando la persona ha una paura generalizzata perché ha mentito. Non è preoccupato per un attacco fisico, ma si prepara lo stesso, a causa delle reali preoccupazioni per il suo benessere dopo aver mentito.

Partendo dall'idea che le persone agiscono come pensano, l'impiego delle esperienze personali di un investigatore e la comprensione di come un criminale deve agire per avere successo è un vantaggio. Dovremmo essere sempre consapevoli che gli agenti cercano $i$ "comportamenti obbligati" del criminale quando elaborano mentalmente una scena del crimine. Il comportamento criminale di un delinquente molto probabilmente assomiglierà ai suoi comportamenti non criminali nella sua vita quotidiana. Non parliamo di un'esatta ripetizione del suo comportamento criminale, ma piuttosto di un comportamento parallelo a quello della sua vita occasionale e lavorativa.

M. Napier, infatti, fornisce delle "perle di saggezza" per un ottimo interrogatorio ad un sospettato:

1. Incoraggia ad utilizzare la tecnica di "interrogatorio ravvicinato" per risolvere affermazioni poco chiare fornite dall'intervistato, suggerimenti sul linguaggio del corpo e per individuare eventuali ambiguità. Una critica ripetuta agli interrogatori della polizia è che esercitano troppi sforzi nella ricerca di bugie piuttosto che nel chiarire aree o argomenti "sensibili" che rivelano informazioni aggiuntive.

2. Viene lanciata una sfida alla tecnica tradizionalmente insegnata agli agenti, nota come "leggere il linguaggio del corpo". L'attuale istruzione su questo argomento è incompleta e la cosa più grave di tutte è che porta a complicazioni, spreco di tempo e risorse in un'indagine, dovuti all'errata selezione e focalizzazione sui sospetti.

3. Durante il colloquio, una persona è invitata e incoraggiata a discutere del suo stile di vita e della sua famiglia tramite domande casuali a risposta aperta, dunque una buona capacità di ascolto rileverà eventuali errori.

4. Ha sottolineato il tratto necessario dell'intervistatore di essere un "attore" abbastanza bravo. Quel talento entra in gioco ripetutamente in interviste e interrogatori condotti correttamente.

5. Ha esortato una revisione speciale di una nuova tattica che ha chiamato "lettura del pensiero" da utilizzare per minare la fiducia di un sospetto nella sua capacità di evitare il rilevamento.

6. L'approccio un po' casuale all'intervista del soggetto è stata modificata tramite la tecnica denominata "intervista del soggetto mirato". L'approccio consiste nell'identificare i punti 
deboli e le vulnerabilità degli obiettivi in specifici crimini violenti utilizzando pratiche "standard" note per ogni tipo di criminale, ad es. assassino, stupratore, molestatore di bambini, stalker, ecc.

7. Ha anche posto un'enfasi speciale sugli aggiustamenti all'uso dei concetti esistenti: selezione dello stile di intervista e di interrogatorio, uso vitale di una conversazione casuale introduttiva, limitare l'attuale dipendenza dalla valutazione dei gesti non verbali nel prendere decisioni significative (specialmente quando si determina chi sospettar) capacità di recitazione necessarie per colloqui e interrogatori efficaci, intervistare sempre prima dell'interrogatorio vero e proprio.

"Il colloquio è il cuore pulsante della divisione investigativa in cui sono in gioco anche vite e proprietà dei soggetti interessati" (Napier M. R., 2020, pag. 31).

Ma attenzione, uno dei più grandi nemici delle indagini e dei procedimenti giudiziari onesti è la tendenza a saltare alle conclusioni senza tutti i fatti. Inoltre per concludere un'indagine in modo positivo gli agenti dovranno tenere consapevolmente aperta la mentalità. Niente metterà un'indagine nello scompiglio più di una mentalità chiusa che di solito si basa su pregiudizi o tecniche investigative improprie.

Inoltre Napier insiste sul dividere l'interrogatorio in una parte iniziale che chiama "intervista" e successivamente passare all'interrogatorio accusatorio vero e proprio (Tab. 1).

Inoltre, l'ormai ex agente speciale dell'FBI, ci elenca le principali caratteristiche che dovrebbe possedere un ottimo agente investigatore per riuscire al meglio a concludere efficacemente un'indagine investigativa:

- Mentalità molto aperta,

- Rimanere sempre calmi, ragionevoli e pratici, senza mai perdere il controllo,

- Comprendere come agisce e pensa il criminale,

- Non essere affrettato saltando subito alle conclusioni,

- Non divulgare troppo informazioni, prima del dovuto,

- Essere sinceri con il sospettato per creare un rapporto di fiducia e collaborazione.

Insomma Michael Napier ha fornito un'ottima spiegazione e interpretazione del suo lavoro, grazie alla sua grande esperienza nell'ambito investigativo e culturale nella formazione di agenti speciali dell'FBI. Con i suoi aneddoti ha descritto perfettamente le tecniche e le strategie di indagine di polizia americana e approfon- dito tutte quelle caratteristiche che un buon investigatore dovrebbe avere per svolgere al meglio il suo lavoro durante un'indagine investigativa.

\section{PNL, strategie di persuasione e manipolazione della comunicazione}

Approfondendo la sfera della comunicazione non verbale è inevitabile imbattersi nelle strategie di persuasione ampiamente utilizzate nell'ambito della Programmazione Neuro-Linguistica (PNL), in inglese neuro-linguistic programming (NLP). Questo è un metodo di comunicazione che cerca di cambiare i pensieri e i comportamenti delle persone per aiutarle a raggiungere i loro obiettivi. Definita da alcuni promotori come approccio alla comunicazione, allo sviluppo personale e alla psicoterapia (Alder H., Heather B., 2007); pensata, ideata e realizzata da Richard Bandler e John Grinder, in California, negli anni '70. Il suo nome suppone una connessione fra i processi neurologici ("neuro"), il linguaggio ("ligustico") e gli schemi comportamentali appresi con l'esperienza ("programmazione"). La PNL deve il suo successo alle idee, alle promesse di successo e alla felicità nel miglioramento del lavoro attraverso lo sviluppo personale. È una disciplina priva di scientificità ed è oggetto di studi in continua evoluzione; quindi al fine di chiarire il suo significato è opportuno specificare e descrivere gli aspetti caratterizzanti della PNL. Gli esperti di PNL insegnano come respirare alla stessa velocità dell'interlocutore, parlare alla stessa velocità o utilizzare lo stesso tono di voce, insomma l'abilità precisa che insegnano durante i corsi è come "ricalcare gli altri". Se un soggetto incontrerà una persona che pensa maggiormente per immagini visive, sarà opportuno descrivere mediante immagini, ma sarà opportuno capire sempre quali siano $i$ momenti migliori per usarle. Sarà l'abilità nel valutare e capire le cose all'esterno che permetterà di distinguere le diverse situazioni e i modi migliori di agire. Da ricordare che al cervello umano "piace" quello che è più simile a noi e impara da ciò che è diverso. L'uomo tende ad imparare tramite le differenze, anche se tende a piacerle ciò che è uguale. Quindi sarà indispensabile prima di tutto, attirare l'attenzione, respirare alla stessa velocità dell'interlocutore, parlare alla stessa velocità o utilizzare lo stesso tono di voce, imitare i movimenti dell'interlocutore e essere alla costante ricerca del consenso. La disciplina viene impiegata per trattare ansie, fobie e stress, in modo

Tab. 1.

Concetti a confronto

Obiettivi

Rappresentazione dell'immagine
dell'agente a beneficio del soggetto
Intervista Interrogatorio

Obiettivi dell'agente a beneficio del soggetto

Dare al soggetto il pieno controllo per raccontare tutte le parti della sua versione degli eventi, inclusi gli alibi e altri fattori attenuanti. Consentito di essere dichiarato senza alcuna interruzione.

Svolge il ruolo di scopritore neutrale e indeciso di tutti i fatti

Ruolo di oratore principale 95\% - 5\% Intervistatore colloca il soggetto nel ruolo di oratore principale e gli trasferisce il $95 \%$ del carico di conversazione

\begin{tabular}{ll} 
Tipi di domande primarie & $\begin{array}{l}\text { Domande aperte utilizzate principalmente } \\
\text { dall'intervistatore. }\end{array}$ \\
\hline Tono di interrogatorio & $\begin{array}{l}\text { L'intervistatore chiede solo per determinare i } \\
\text { dettagli del crimine e il ruolo dell'intervistato }\end{array}$ \\
\hline
\end{tabular}

Passare dal formato dell'intervista o della conversazione casuale al confronto per ottenere chiarimenti sulla storia. Ottenere ammissioni/confessioni.

Il suo ruolo è quello di essere inequivocabilmente convinto della colpevolezza del soggetto e di accusarlo fermamente.

L'interrogatore assume il ruolo di porre domande, fare dichiarazioni e persuaderlo a discutere $\mathrm{i}$ suoi atti criminali. Il $95 \%$ del ruolo di parola va all'interrogatore.

Utilizza tutti i tipi di domande legali.

L'interrogatore fa un'accusa. È fermamente convinto della colpevolezza del soggetto 
da migliorare le risposte emotive in determinate situazioni, raggiungere obiettivi personali di successo, rimuovere pensieri e sentimenti negativi associati ad eventi del passato e migliorare le proprie capacità comunicative. Perciò, la PNL è utilizzata per lo sviluppo individuale, facendo leva sul "potenziamento" delle proprie capacità con l'obiettivo di aumentare la fiducia personale e le proprie doti comunicative. La PNL non è una "cosa" che si usa; è un mezzo per imparare a gestire il cervello umano in ogni momento, perché, se gestissimo il cervello in ogni momento, non avremmo molti problemi da risolvere (Bandler R., La Valle J., 2003).

"È una terapia alternativa che si pone l'obiettivo di educare le persone alla conoscenza di sé, alla comunicazione efficace, al cambiamento degli schemi mentali e dei comportamenti emotivi". Essa, dunque, è considerata una tecnica di persuasione nella branca delle pseudoscienze. Non ha lo scopo di manipolare il beneficiario, bensì quello di creare nuovi canali comunicativi e di far confluire a proprio vantaggio i processi naturali delle persone.

In altri casi, nei corsi di PNL, è possibile riflettere i comportamenti del proprio interlocutore come se fosse allo specchio, oppure allineare la propria fisiologia con quella del parlante, cioè se lui clicca la penna su e giù, è possibile per il praticante battere il piede su e giù a ritmo con l'interlocutore.

Analizzare e studiare quali siano le strategie di persuasione e le tecniche che portano le persone ad acconsentire alle richieste non è semplice; ma soprattutto quelle più utilizzate sono riconoscibili osservando i professionisti che sfruttano questi aspetti a loro vantaggio. Durante la giornata con amici, parenti, vicini di casa, tutti li utilizzano e ne sono vittima, ma semplicemente non se ne rendono conto. Solo i professionisti - venditori, pubblicitari, esattori e così via - hanno consapevolezza di cosa possa funzionare o no utilizzando le "armi della persuasione" (Cialdini R., 1989). Il potenziale di queste strategie è straordinario, ma poiché esistono migliaia di tecniche, per semplificarne e capirne l'utilità, sono state suddivise in sei categorie, ognuna delle quali corrisponde ad un principio piscologico di base: reciprocità, impegno/coerenza, riprova sociale, simpatia, autorità e scarsità.

Il comportamento umano varia a seconda delle situazioni, emozioni ed esperienze che un soggetto sperimenta nella vita, ma in alcuni contesti può capitare che il comportamento, che viene messo in atto, non sia adatto alla situazione. Non sempre il comportamento stereotipato o informale è il modo giusto di agire e in mancanza di quei criteri sommari, che aiutano a gestire le situazioni; è possibile rimane paralizzati a cercare il modo migliore di procedere, perdendo l'occasione che si prospetta. L'uomo è un essere vulnerabile e per rendersene conto basta osservare il comportamento animale quando gli animali imitano i comportamenti di altre specie per trarre in inganno i predatori o le loro vittime. Lo stesso accade nella "giungla umana", dove gli approfittatori, imitano alla perfezione i segnali attivatori che mettono in moto le risposte automatiche dei soggetti. I programmi automatici spesso sono comportamenti psicologici e stereotipati messi in pratica perché imparati e accettati nell'esperienza di vita. Ma ci sono persone che sanno bene come sfruttare e manipolare questi segnali a loro vantaggio senza il minimo sforzo. Insomma, la società progredisce estendendo il numero di operazioni che un soggetto possa eseguire senza pensarci e che lo rendono terribilmente vulnerabile di fronte a chiunque ne conosca il funzionamento (Cialdini R., 1989).

In una versione generale, la manipolazione è l'operazione di elaborazione e diffusione di messaggi volta, deliberatamente, a influenzare in modo intenzionale e in modo moralmente scorretto un determinato obiettivo; così i destinatari eluderanno le loro capacità critiche, alterando $i$ fatti che sono oggetto dell'informazione. "In termini pragmatici, la manipolazione è una sottocategoria della comunicazione" (Watzlawich P., 1976). Se, come sosteneva
Watzlawich "comunicare è sinonimo di influenzare", manipolare è sinonimo di influenzare approfondendo dei "punti sensibili". Perciò, affinché ci sia manipolazione, deve sussistere una relazione asimmetrica tra chi agisce e il soggetto o i soggetti scelti.

"Nelle relazioni umane le strategie di manipolazioni sono in realtà molto comuni e chi le mette in atto è di solito un individuo psicologicamente disturbato, bisognoso di persone da controllare in qualche modo (anche se questi non sono propriamente i soggetti più idonei a diventare esperti della manipolazione)" (Sperzagni C. 2020). Nel contesto della manipolazione professionale, invece, ci sarà uno dei due individui che deliberatamente mette in atto tecniche e strategie per sottoporre l'altro in una situazione che non può sopportare e controllare (manipolazione psicologica). Ma la manipolazione assume una portata ancora più ampia quando si accosta alla proposta sistematica di stereotipi e di visioni distorte della realtà o comunque quando i messaggi esercitano un grado di influenza inavvertita sui ricettori dell'azione comunicativa. Essa può assumere un secondo significato riguardo la sfera della comunicazione di massa, per cui si tende a sottovalutare il ruolo svolto dal ricettore del messaggio e si ipotizza, nei processi di influenza dei mass media, una situazione squilibrata a favore dell'emittente. Nell'idea marxista infatti i mass media sono dei veri e propri "mezzi" di manipolazione: chi possiede o controlla i mezzi della produzione materiale, possiede e controlla allo stesso tempo i mezzi della produzione intellettuale e influenza le idee di coloro che con essi è possibile raggiungere (Lever F., Rivoltella P.C., Zanacchi A., 2020). In quest'ottica entra anche la questione della propaganda definita come una forma di comunicazione destinata a diffondere nel pubblico la conoscenza di caratteristiche, idee, obiettivi, programmi, azioni, servizi, prodotto, ecc.; di gruppi, associazioni, organizzazione, istituzioni operanti nei diversi ambiti - sociale, politico, economico, culturale, religioso - della società. Ma con questo termine si può far riferimento anche alle finalità persecutorie insite nel termine. In questa accezione, la propaganda è l'insieme di forme di comunicazione e di attività promozionali messe in atto per esercitare un'influenza mirata. Tale influenza può ritenersi efficace sia quando determina il rafforzamento o la convenzione di opinioni, atteggiamenti e comportamenti; sia quando induce a nuove opinioni, idee, atteggiamenti e comportamenti. Tuttavia, la propaganda finalizzata alla persuasione diviene espressione di un progetto ideato e realizzato, con l'impiego di specifiche competenze e professionalità, solo nelle moderne società industria$\mathrm{li}$; in relazione alla necessità di raggiungere un pubblico di vaste dimensioni e alla possibilità di utilizzare come canali prevalenti i mezzi di comunicazione di massa. In definitiva alla propaganda vengono attribuite funzioni diverse: una funzione informativa al servizio del destinatario, valutata positivamente per la diffusione di elementi di conoscenza, e una funzione manipolatoria al servizio della fonte da cui parte, che viene valutata negativamente, in quanto strumento di persuasione.

La forma di manipolazione che oggi come oggi ci tocca di più è quella digitale. La comunicazione digitale è l'insieme di tutte le attività (visive, audiovisive, testuali, ecc.) che vengono condivise attraverso un sistema di tecnologia digitale ovvero tramite le applicazioni (social network, e-mail, forum, ecc.), i siti web/blog, piattaforme digitali, ecc., che siano raggiungibili al più alto numero di persone possibile. Essa è molto importante perché permette a chiunque, anche un piccolo imprenditore o libero professionista, di poter veicolare, comunicare il proprio business ad un "target" ben specifico, questo perché i contenuti prodotti e condivisi digitalmente sono altamente personalizzabili. Internet ha modificato e rivoluzionato la comunicazione in tutti i settori, dalla politica alla pubblicità; approcciandosi in maniera positiva, cercando di sfruttare la forza, le caratteristiche e le ultime tecnologie; cosa indispen- 
sabile per rimanere competitivi ad ogni livello. Comunicare, oggi, significa raggiungere il più velocemente possibile un pubblico; ed è questo l'obiettivo della comunicazione digitale. La sfida comunicativa si gioca sul "come comunicare" e non più su "cosa comunicare"; conta di più il mezzo, il canale, il medium utilizzato, piuttosto che il contesto in senso stretto. E la comunicazione digitale è il mezzo più immediato: in media, ciascuno di noi, trascorre 6 ore e 42 minuti davanti ad uno smartphone o un computer navigando sul web. Ecco perché questo canale è diventato il canale centrale nelle strategie comunicative e informative. L'immediatezza che ha contribuito enormemente allo sviluppo dei social network, non si incarna però solo nella velocità della parola. Con Twitter, Facebook o Instagram, una buona dose di contenuti e quindi di messaggi, non si manifesta tramite un testo scritto, ma tramite un'immagine o addirittura un video.

Con lo sviluppo della tecnologia sociale contemporanea, assistiamo ad un nuovo fenomeno: 1" "inquinamento" delle informazioni su scala globale. I suoi impatti diretti e indiretti sono difficili da quantificare, ma le implicazioni a lungo termine delle campagne di disinformazione sono le più preoccupanti. Una complessa rete di motivazioni per creare, diffondere e consumare messaggi "inquinati", una miriade di tipi e tecniche per amplificare i contenuti, innumerevoli piattaforme che ospitano e riproducono questi contenuti; e la velocità vertiginosa della comunicazione che riesce ad influenzare chiunque in ogni angolo del mondo. Gli impatti diretti e indiretti dell'inquinamento delle informazioni sono difficili da quantificare, essendo un fenomeno recente, ma siamo solo alla prima delle fasi di comprensione delle loro implicazioni. Dai risultati del voto "Brexit" nel Regno Unito, la vittoria di Donald Trump negli Stati Uniti, per fare celebri esempi; si è discusso molto di come il disturbo dell'informazione stia influenzando le democrazie di tutto il mondo. Più preoccupanti, tuttavia, sono le implicazioni a lungo termine della disinformazione e delle conseguenti campagne progettate specificamente per seminare sfiducia e confusione e per acutizzare le divisioni socioculturali esistenti, utilizzando tensioni nazionalistiche, etniche, razziali e religiose. Per affrontare efficacemente i problemi di cattiva informazione, i punti su cui lavorare dovranno essere: pensare in modo più critico alla lingua che usiamo per cogliere la complessità del fenomeno; investigare adeguatamente le implicazioni per la democrazia quando sussistono informazioni false o fuorvianti che circolano on-line; chiarire il potere dei mass media e in particolare della televisione e dei social network nella diffusione e amplificazione di informazioni di scarsa qualità; capire l'impatto che il giornalismo ha avuto nella diffusione della disinformazione. Insomma, indagare tutti quei fenomeni che possono influenzare e favorire campagne disinformative.

Il mondo della disinformazione è una caratteristica fondamentale nello spionaggio o intelligence militare. Il compito principale dei servizi segreti è duplice: raccogliere informazioni sul nemico (spionaggio) e impedire a quest'ultimo di raccogliere informazioni su di essi (controspionaggio). Esiste un terzo compito che è quello di fornire false informazioni al nemico, circa i piani di un determinato servizio, traendo in inganno l'avversario. Azioni che sono quindi "giochi d'inganno", di disinformazione, ma il punto è quello di far pensare la cosa sbagliata al nemico e di non renderlo consapevole delle sue supposizioni erronee fino a quando non è troppo tardi. Quindi, nel lavoro dei servizi segreti, ma come in qualsiasi altro contesto di comunicazione, l'attendibilità dell'informazione dipende da due fattori: la probabilità dell'informazione stessa e la credibilità della sua fonte (Watzlawich P., 1976, pag. 120). Perciò, possiamo affermare, dal punto di vista della comunicazione che è interessante come questi contesti si presentino unicamente quando vengono capovolte le regole normali della comunicazione e la disinformazione diventa l'ultima meta e vetta da raggiungere.
Solo ragionando e capendo quali siano i limiti che l'uomo può raggiungere che riusciremo a mettere in atto comportamenti e azioni ragionate e sensate. Se l'individuo si farà sovrastare dalle emozioni, la ragione finisce per diventare istinto, iniziando a compiere errori che possono essere sfruttati da chi ha interesse di ingannarci.

\section{Conclusioni}

In sede di bilancio conclusivo, il dato più evidente emerso dal presente lavoro è l'adattamento alla mancanza di una definizione vera e propria di comunicazione. Per cui la riflessione si è incentrata proprio sull'importanza del fenomeno comunicativo e sulle sue varie caratteristiche per carpirne l'essenza. Infatti si sono trattate le varie strutture e funzioni che il fenomeno comunicativo può rappresentare e influenzare al tempo stesso, minando anche la sfera investigativa e criminologica. L'analisi compiuta ha assunto necessariamente punti di vista diversi, inquadrato aspetti parziali del fenomeno, prodotto declinazioni particolari e specifiche per definire un termine così ampio e variegato e in ultima battuta fornito un contributo analitico sui fenomeni socio-psico-politici che intervengono e influenzano il processo comunicativo.

\section{Bibliografia}

Acquaviva M., (2020), Interrogatorio penale: come funziona? https://www.laleggepertutti.it/

Aga I. N., (2000), L'arte di non lasciarsi manipolare, Paoline Editoriale, Milano.

Alder H., Heather B., (2007), PNL in 21 giorni. Un approccio rivoluzionario alla comunicazione umana e allo sviluppo personale, Il Punto d'Incontro, Vicenza.

Anolli L., (2002), Psicologia della comunicazione, Il Mulino, Bologna.

Anolli L., (2006), Fondamenti di psicologia della comunicazione, Il Mulino, Bologna.

Argyle M., (1978), Comunicazione non verbale, Zanichelli, Bologna.

Bandler R., La Valle J., (2003), PNL per la persuasione. Come la programmazione neuro-linguistica può aumentare le tue vendite, Alessio Roberti Editore Srl, Bergamo.

Baratta P., Sini F., Trupia, Santucci, Fele, Balabbio, Mizzau, Saravai, Trevi, (1993) Manipolazione, Anabasi.

Barbier A. (2020), Relazioni tossiche. Violenza psicologica e manipolazione emotiva, tratto da https://www.psicoterapiapersona.it/

Benini A., (2013), Effetto Milgram: un falso? Il Sole 24 Ore, Numero 356

Bernays E., (2018), Propaganda. L'arte di manipolare l'opinione pubblica, Piano B, Prato.

Bonadonna R., (2018), CNV: Il linguaggio analitico della comunicazione - il capo. https://www.mediagold.it/magazine/gliesperti/cnv-comunicazione-non-verbale/cnv-il-linguaggio-analogico-della-comunicazione-il

Bonaiuto M, Maricchiolo F., (2003), La comunicazione non verbale, Carocci editore, Roma.

Bracchi R., (2020), La comunicazione (etimologia). https://www. lacomunicazione.it/voce/comunicazione-etimologia/

Chomsky N., Herman E. S., (2008) La fabbrica del consenso. La politica e i mass media. Il Saggiatore, Milano.

Cialdini R., (1989), Le armi della persuasione. Come e perché si finisce col dire sì, Giunti Editore S.p.a, Firenze. 
Concas A., (2014), L'interrogatorio dell'indagato durante le indagini, Referente Aree Diritto Civile, Commerciale e Fallimentare e Diritto di Famiglia, Cagliari.

Corona S., (2016), Il Milton model. https://www.samuelecorona. com/il-milton-model/

D’Auria S., (2006), Cesare Lombroso, gli studi ed i "successori” del grande antropologo, Rassegna penitenziaria e criminologica, 10:1-51.

D’Auria S. (2020), Cesare Lombroso. Gli studi ed i "successori” del grande antropologo. http://www.rassegnapenitenziaria.it/

Darley J. M., Latané B., (1968), Journal of Personality and Social Psychology, vol. 8.

Darwin C., (2012), L'espressione delle emozioni nell'uomo e negli animali, Bollati Boringhieri, Torino.

De Lalla G. (2013), L'interrogatorio dell'indagato e la difesa. https:// www.studiolegaledelalla.it/interrogatorio-indagato-difesa/

Delladio D., (2020), Wilbur Schramm - Modelli e campi d'esperienza. https://www.loomenstudio.com/wilbur-schramm/

Della Luna M., Cioni P., (2009), Neuroschiavi. Liberiamoci dalla manipolazione psicologica, politica, economica e religiosa, Macro Edizioni, Diegaro di Cesena.

Demarco M., (2020), Lombroso, il suo lato poetico. https:/www.c orriere.it/cultura/17_dicembre_06/cesare-lombroso-criminologo-lettere-museo-torino

Diadori P. (2013), Gestualità e didattica della seconda lingua: questioni interculturali, in Borello P.E., Luise M.C., Aspetti comunicativi e interculturali nell'insegnamento delle lingue, Edizioni dell'Orso, Alessandria. http://www.siena-art.com/diadori/Testi/ 08iGESTI.pdf

Diamantopoulos G., Woolley S., Spann, M., (2009), A Critical Review of Past Research into the Neuro-linguistic Programming Eye-accessing Cues Model, Current Research in NLP.

Ekman P., Friesen W., (2003), Giù la maschera. Come riconoscere le emozioni dell'espressione del viso, Giunti, Firenze.

Emerson R.W., (2003), Fiducia in sé stessi, Ibis, Como.

Fabris F., (2017), Dalla Scuola di Palo Alto, la pragmatica della comunicazione umana, Milano.

Ferri E., (1883), La Scuola positiva di diritto criminale, Prelezione al corso di diritto e procedura penale nella R. Università di Siena pronunciata il 18 Novembre 1882, Altro, Enrico Torrini, Siena.

Fiore F., (2018), Paul Ekman e i suoi importanti studi sull'universalità delle emozioni e delle espressioni facciali. https://www.stateofmind.it/2018/06/paul-ekman-emozioni-espressioni-facciali/

Gerbner G., (1973), Communications Technology and Social Policy: Understanding the New "Cultural Revolution, Interscience Publication, New York.

Horkheimer M., Adorno T. W., (2010), Dialettica dell'illuminismo, Einaudi, Torino.

Kendon A., (1970), Movement coordination in social interaction: Some examples described. Acta Psychologica, Amsterdam.

Kendon A., (2004), Gesture: visible action as utterance, Cambridge University Press.

Kunz, P. R., \& Woolcott, M. (1976). Season's greetings: From my status to yours, Social Science Research.

Leahey R. E., Lewin R., (1992), Origins Reconsidered: In Search of what Makes Us Human, Doudleaday.

Lever F., Rivoltella P.C., Zanacchi A. (2020), La comunicazione. Dizionario di scienze e tecniche. https://www. lacomunicazione.it/

Lombroso C., (1876), L'uomo delinquente. In rapporto all'antropologia, alla giurisprudenza ed alla psichiatria, Fratelli Bocca Editori, Torino.

Losito G., (1998), Un'introduzione alla teoria e alla ricerca sociale, Carocci, Roma.
Marchetti P., (2008), L'armata del crimine. Teoria e repressione della recidiva in Italia. Una genealogia, Cattedrale Editore, Ancona.

Maricchiolo F. (2017), La comunicazione non verbale. Caratteristiche e funzioni. http://www.it.in-mind.org/article/lacomunicazione-non-verbale-caratteristiche-e-funzioni/

Mastronardi V., (2005), Il profilo criminologico. Dalla scena del crimine ai profili socio-psicologici, Giuffrè Editore, Milano.

Mastronardi V., (2007), Manuale di comunicazione non verbale, Carrocci Faber editore, Roma.

McLuhan M., (1995), Gli strumenti del comunicare, Il Saggiatore, Milano.

Micheletti S., (2016), Cos'è la PNL? Capiamolo una volta per tutte. http:/www.pnlpedia.com/cos-e-la-pnl/

Milgram S., (2007), Obbedienza all'autorità: uno sguardo sperimentale, HarperCollins, Milano.

Molden D., (2011), How to Manage With NLP, Pearson Financial Times.

Montelli A., (2020), PNL: cos'è e a cosa serve la programmazione neuro-linguistica. https://www.starbene.it/benessere/corpomente/pnl-programmazione-neurolinguistica-cos-e/

Morris D., (1978), L'uomo e i suoi gesti, Mondadori, Milano.

Morris D., (1982), The Soccer Tribe, Mondadori, Milano.

Negretto C., (2010), Robert Cialdini: le armi della persuasione, Milano.

Pacori M., (2011), La neurologia della CNV. https:/www.linguaggiodelcorpo.it/2011/10/20/la-neurologia-della-cnv/

Pacori M., (2016), Come interpretare i messaggi del corpo, Giunti, Milano.

Pio E. Ricci Bitti, Bruna Zani, (1983), La comunicazione come processo sociale, Il Mulino, Bologna.

Purayidathil T., (2020), La storia della comunicazione. https:// www.lacomunicazione.it/voce/storia-della-comunicazione/

Regan D.T., (1971), Effects of a Favor and Liking on Compliance. Jornal of experimental social psychology, Cornell University.

Sacks H., Schegloff E., Jefferson G., (1974), A simplest systematics for the organisation of turn taking for conversation, Language.

Schiaralli E., (2013), Quante volte si sentono dire frasi del genere "quello ha una faccia da delinquente" oppure "è un tipo che non mi piace"? Corato.

Stern R., (2009), “Come mi vuoi?”' Imparare a difendersi dalla manipolazione emotiva, Edizioni Corbaccio, Milano.

Stern R., (2011), Non mi puoi manipolare. Riconoscere e difendersi dagli abusi emotivi, TEA, Milano.

Valko K. (2018), La definizione di comunicazione non verbale, in Linguaggio del corpo. https://www.igorvitale.org/

Volli U., (1994), Il libro della comunicazione, Il Saggiatore, Milano.

Wardle C., (2017), Information Disorder: Toward an interdisciplinary framework for research and policy making, Council of Europe report DGI.

Watzlawick P., (1976), La realtà della realtà. Confusione, disinformazione, comunicazione, Astrolabio, Ubaldini Editore, Roma.

Watzlawick P. (1976), Pragmatica della comunicazione umana, Astrolabio, Milano.

Williams F.P., McShane M.D., (2002), Devianza e criminalità, il Mulino, Bologna.

\section{Sitografia}

http://www.ancrim.it/come-si-diventa-criminologi-e-criminalisti. $\mathrm{html}$

http://www.internationalsecurityinterest.com/

https://www.brocardi.it/dizionario/3695.html 
https://initalia.virgilio.it/il-museo-degli-orrori-di-lombroso-e-la-triste-storia-del-teschio-di-villella/

https://www.inventati.org/emiliaparanoica/comunicazione.html https://lab-ncs.com/

https://lombrosoproject.unito.it/list.php/

https://psycnet.apa.org/search/basic?id=fd55ca6a-e7a0-4869-c145$36 \mathrm{fe} 14 \mathrm{dcc} 8 \mathrm{~d} 8$

https://socialnetworkabc.wordpress.com/1-3-funzioni-della-comunicazione-jakobson/

https://sviluppo-personale.com/metamodello-della-pnl/

https://unaparolaalgiorno.it/significato/comunicare

https://wearesocial.com/

https://www.corriere.it/salute/dizionario/antropologia criminale/ind

ex.shtml?refresh ce-cp/

https://www.humanitas.it/malattie/epilessia/ https://www.ilmessaggero.it/primopiano/cronaca/cesare_lombroso/ https://www.ilmessaggero.it/primopiano/cronaca/cesare lombro-

so_web_sito_delinquenza_universita_delinquenza-3836477. html

https://www.internationalsecurityinterest.com/

https://www.lastampa.it/

https://www.mininterno.ne/

https://www.pensieriparole.it/

https://www.stateofmind.it/tag/criminologia/

https://www.thismarketerslife.it/

https://www.treccani.it/enciclopedia/io/

https://www.treccani.it/vocabolario/prossemica/

https:/www.unicomunicazione.it/pnl-cose-la-programmazioneneuro-linguistica/
Correspondence: Noemi Martini.

E-mail: mariaerikacravotta@yahoo.it

Key words: communication; NVC; NLP; persuasion

Parole chiave: comunicazione; CNV; PNL; persuasione.

Palabras clave: comunicación; CNV; PNL; persuasión.

Received for publication: 29 May 2020.

Accepted for publication: 26 June 2020.

This article is distributed under the terms of the Creative Commons Attribution Noncommercial License (by-nc 4.0) which permits any noncommercial use, distribution, and reproduction in any medium, provided the original author(s) and source are credited.

${ }^{\circ}$ Copyright: the Author(s), 2020

Licensee PAGEPress, Italy

Rivista di Psicopatologia Forense, Medicina Legale, Criminologia $2020: 25: 547$

doi:10.4081/psyco.2020.547 